\title{
Add-On Effect of Chinese Herbal Medicine on Mortality in Myocardial Infarction: Systematic Review and Meta-Analysis of Randomized Controlled Trials
}

\author{
Vincent C. H. Chung, ${ }^{1}$ Mao Chen, ${ }^{1}$ Qin Ying, ${ }^{1}$ Wilson W. S. Tam, ${ }^{1}$ Xin Yin Wu, \\ Polly H. X. Ma, ${ }^{1}$ Eric T. C. Ziea, ${ }^{2}$ Vivian C. W. Wong, ${ }^{2}$ and Jin Ling Tang ${ }^{1}$ \\ ${ }^{1}$ The Jockey Club School of Public Health and Primary Care, The Chinese University of Hong Kong, Hong Kong \\ ${ }^{2}$ Chinese Medicine Department, Hong Kong Hospital Authority, Hong Kong \\ Correspondence should be addressed to Jin Ling Tang; jltang@cuhk.edu.hk
}

Received 31 August 2012; Accepted 16 November 2012

Academic Editor: Vassya Bankova

Copyright (C) 2013 Vincent C. H. Chung et al. This is an open access article distributed under the Creative Commons Attribution License, which permits unrestricted use, distribution, and reproduction in any medium, provided the original work is properly cited.

In China, Chinese herbal medicine (CHM) is widely used as an adjunct to biomedicine (BM) in treating myocardial infarction (MI). This meta-analysis of RCTs evaluated the efficacy of combined CHM-BM in the treatment of MI, compared to BM alone. Sixty-five RCTs (12,022 patients) of moderate quality were identified. 6,036 patients were given CHM plus BM, and 5,986 patients used BM only. Combined results showed clear additional effect of CHM-BM treatment in reducing all-cause mortality (relative risk reduction $\left.(\mathrm{RRR})=37 \%, 95 \% \mathrm{CI}=28 \%-45 \%, I^{2}=0.0 \%\right)$ and mortality of cardiac origin $(\mathrm{RRR}=39 \%, 95 \% \mathrm{CI}=22 \%-52 \%$, $I^{2}=22.8$ ). Benefits remained after random-effect trim and fill adjustment for publication bias (adjusted RRR for all-cause mortality $=29 \%, 95 \% \mathrm{CI}=16 \%-40 \%$; adjusted RRR for cardiac death $=32 \%, 95 \% \mathrm{CI}=15 \%-46 \%)$. CHM is also found to be efficacious in lowering the risk of fatal and nonfatal cardiogenic shock, cardiac arrhythmia, myocardial reinfarction, heart failure, angina, and occurrence of total heart events. In conclusion, addition of CHM is very likely to be able to improve survival of MI patients who are already receiving BM. Further confirmatory evaluation via large blinded randomized trials is warranted.

\section{Background}

1.1. Myocardial Infarction: Disease Burden and Therapeutic Options. Incoronary artery disease, a critical reduction of the blood supply to the heart may result in myocardial infarction (MI), a phenomenon owing to the formation of an area of necrosis in heart muscles caused by inadequate supply of blood to the muscles, usually as a result of occlusion of a coronary artery. About a quarter of MI patients will die from it due to complications including cardiogenic shock, cardiac perforation, embolism, heart failure, papillary muscle rapture, rhythm disturbances, or autoimmune pericarditis. Current evidence on biomedicine (BM) treatment suggests that aspirin, thrombolytics with or without adding lowmolecular-weight heparin, beta-blockers, ACE inhibitors, and nitrates are beneficial for improving outcomes in people with MI. Invasive procedures including coronary artery bypass grafting (CABG) and percutaneous transluminal coronary angioplasty (PTCA, balloon angioplasty) were also found to be useful. However, their efficacy in preventing death is not without limitations. For instance, beta-blockers have no short-term effect on mortality, and they may increase the risk of cardiogenic shock. Thrombolytics may cause stroke and major bleeding while reducing mortality, and those who are treated will receive no additional benefits from nitrates [1].

Despite these therapeutic advances, coronary artery disease remained to be the foremost leading cause of death in both low- and middle income countries as well as high-income countries, contributed $11.8 \%$ and $17.3 \%$ of total deaths, respectively [2]. Researchers are evaluating the potential benefits and harms of add-on treatments like 
vasodilators and positive inotropes on mortality [3]. Chinese herbal medicine (CHM) is another novel candidate as an add-on treatment.

1.2. Chinese Herbal Medicine for Treating Myocardial Infarction. In China, CHM is widely prescribed in both outpatient and inpatient settings [4]. Amongst community health clinics, $75 \%$ provide both BM and traditional Chinese medicine (TCM) treatments. TCM hospitals comprised $13.8 \%$ of all hospitals, and $90 \%$ of the BM hospitals are annexed with TCM departments [5]. Given the omnipresence of TCM services within the Chinese healthcare system, it is not uncommon for clinicians to prescribe $\mathrm{CHM}$ as an adjunct to BM treatment in the management of potentially lifethreatening conditions including MI [6]. One of the most researched single herbs is Radix Astragali, which exerts its therapeutic effectiveness by inhibiting cardiac fibrosis, reducing infarct size, and increasing capillary and arteriole densities [7]. Commonly used Chinese proprietary medicines include Shexiangbaoxin tablets and Tongxinluo capsules. Shexiangbaoxin tablets are found to slow MI pathogenesis by inhibiting hypertrophy related metabolites [8]. On the other hand, Tongxinluo capsules act by promoting local blood supply and thus limit infarct size [9]. CHM injections based on sheng mai san are also widely prescribed. It reduces infarct size via the activation of protein kinase $C$, opening of the mitochondrial KATP channels, and lowering the concentration of 5-hydroxytryptamine, norepinephrine, methionine-enkephalin, and leucine-enkephalin $[10,11]$.

\subsection{Synthesizing Chinese Herbal Medicine Trials: Focusing} on Objective Outcomes. The average effect of these CHM formulae as an adjunct to BM could be estimated using random effect meta-analyses of randomized controlled trials (RCTs) [12]. One of the major caveats in conducting systematic reviews on CHM is that existing RCTs are often prone to high risks of bias, thus limiting their usefulness in elucidating treatment effectiveness [13]. However, results from a recent metaepidemiological study have provided an alternative perspective on this issue. It is suggested that objective outcomes are less susceptible to bias associated with inadequate allocation concealment and blinding [14, 15]. Accordingly, by focusing on objective outcomes like mortality, we may partially overcome limitations imposed by the relatively high risk of bias amongst CHM trials.

1.4. Aim of This Paper. Taking into account the methodological considerations above, we performed a systematic review and meta-analysis of RCTs on the efficacy and safety of CHM for MI as an add-on to BM treatment, with a focus on objective critical outcomes including death, recurrent myocardial infarction, and other post-MI cardiac consequences.

\section{Methods}

2.1. Criteria for Considering Studies for This Paper. We included RCTs comparing the efficacy and safety of CHM plus $\mathrm{BM}$ versus $\mathrm{BM}$ alone. $\mathrm{CHM}$ is defined as any preparation containing at least one herb or its extraction referenced in the 2010 Chinese Pharmacopeia [16]. We included RCTs which enrolled adult MI patients regardless of gender, age, ethnicity, or comorbidities. We focused on the primary outcomes of (i) mortality of cardiac origin and (ii) all-cause morality. We also consider the following as secondary outcomes: (i) recurrence of MI and (ii) other nonfatal, post-MI cardiac outcomes including cardiac arrhythmia, heart failure, cardiac rupture, cardiogenic shock, and angina. Adverse events reported by authors were also summarized. We imposed no restrictions on language and publication status.

2.2. Search Methods for Identification of Studies. We searched 8 electronic databases since their inception to July 2010, including CENTRAL, MEDLINE, EMBASE, CINAHL, AMED, Chinese Biomedical Database (CBM), Chinese Medical Current Contents (CMCC), and Traditional Chinese Medical Literature Analysis and Retrieval System (TCMLARS) (Figure 1). Search strategies are shown in Appendix 1 in the Supplementary Materials available online at http://dx.doi.org/10.1155/2013/675906.

\subsection{Data Collection and Analysis}

2.3.1. Selection of Studies, Data Extraction, and Risk of Bias Assessment. Two reviewers (Y. Qin and C. Mao) independently screened the titles and abstracts to assess their eligibility. Full texts of potentially eligible citations were retrieved for detailed examination. Selection discrepancies were settled through discussions between these two authors. The remaining disagreements were resolved by consulting another author (J. L. Tang). For included RCTs, comprehensive information on patients, CHM interventions, and baseline and control treatments, as well as outcomes, was extracted. Risks of bias amongst included RCTs were evaluated by the Cochrane collaboration's risk of bias assessment tool [17]. The assessment composed of a description and a judgement for each entry in a risk of bias table, including (i) sequence generation, (ii) allocation sequence concealment, (iii) incomplete outcome data, (iv) selective outcome reporting, and (v) other potential sources of bias. Blinding was assessed for the primary outcome of all-cause morality.

2.3.2. Data Analysis. Analyses were conducted using Stata 11 and R software. Dichotomous efficacy outcomes were expressed as relative risk reduction (RRR) and relative risk (RR), while $\mathrm{RR}$ was used for adverse events. 95\% confidence intervals (CIs) were calculated for all estimates. We performed random-effect meta-analysis separately for each outcome. For primary outcomes of all-cause mortality and cardiac death, funnel plots were drawn for assessing publication bias. In case of asymmetry, random trim and fill analysis were performed as a sensitivity analysis [18]. Tests for heterogeneity were performed with chi-squared testes, at a significance level of $P=0.1 . I^{2}$ statistic was calculated to estimate variation across studies. We regarded $I^{2}<25 \%$ as an indicator of low heterogeneity level, $25-50 \%$ as moderate level, and higher than $50 \%$ as high level [19]. Heterogeneity 


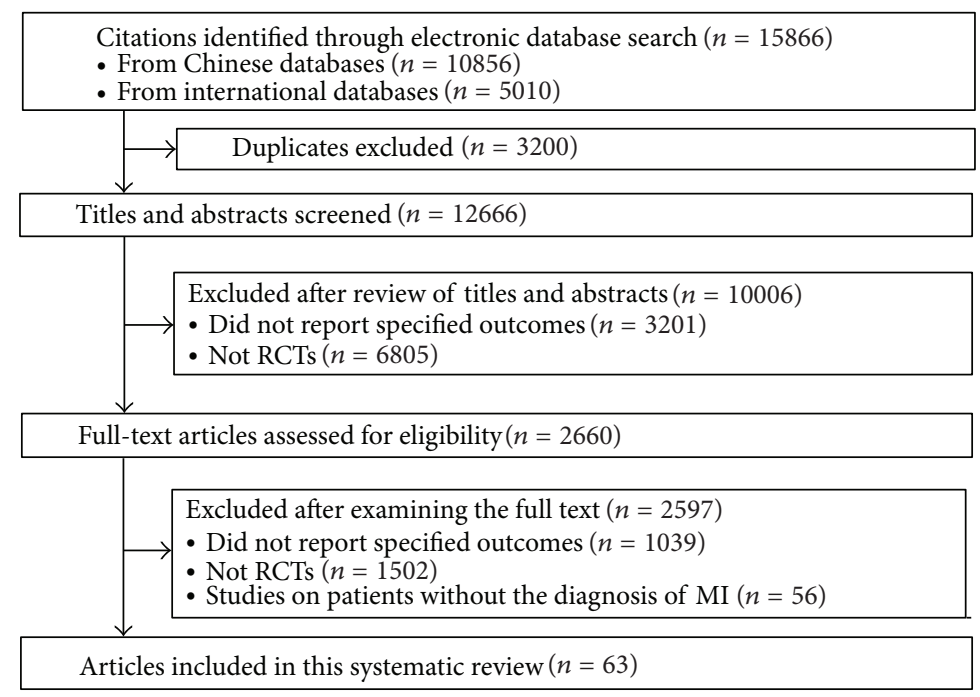

FIGURE 1: Flow chart of literature search and study selection.

was explored with random-effect metaregression using baseline risk, mean age, route of drug administration (oral versus intravenous), and treatment duration as covariates, taking into account the sample size requirement of including not more than 1 covariate for every 10 studies [20]. We expected that higher baseline risk and mean age could be associated with a smaller effect [1], while intravenous administration and longer treatment duration could be associated with a larger effect.

\section{Results}

3.1. Literature Search. As shown in Figure 1, our search in electronic bibliographical databases yielded 12,666 citations after removal of duplications, of which 2,660 were classified as potentially relevant and were subjected to a full-text assessment. A total of 65 RCTs published in 63 articles met the inclusion criteria. Details of these studies are presented in Table 1.

3.2. Study Characteristics. A total of 6,036 patients were enrolled in the CHM plus BM group, and 5,986 patients were allocated to the BM only group. The average size of the trials was 185 participants (ranging from 28 to 2735 participants per trial). Fifty trials reported treatment duration and the average duration was 68.9 days, ranging from 3 to 1440 days. Forty-nine trials reported the length of followup. The average follow-up length was 7.1 months, ranging from 0.1 to 84 months.

For diagnostic criteria, $36(55.4 \%)$ studies applied the 1979 World Health Organization criteria, which enrolled patients with at least two of the following three presentations: chest pain or discomfort, an elevation in CK-MB levels, or an ECG with significant ST-segment elevations [84]. Four adopted criteria from the Chinese Society of Cardiology [85] and one used criteria from the European Society of Cardiology [86]. Twelve applied author-defined diagnostic criteria, and the remaining 12 did not report criteria used.
Thirty-one standardized Chinese herbal formulae were examined in $63(96.9 \%)$ of the 65 included studies, while the other two studies used an individualized approach. 32 (50.0\%) preparations were administrated orally, 30 (46.9\%) were prescribed as herbal injections, and 2 (3.12\%) trials used both intravenous and oral treatments. Eight formulae were evaluated by three or more trials. In total, these formulae were assessed in 38 studies, constituting $58.5 \%$ of all included trials.

(i) Nine (13.8\%) trials studied Shenmai injection, which contains ginsenoside, ginseng polysaccharide, Ophiopogon polysaccharides, and Ophiopogon flavonoids extracted from Panax ginseng and Ophiopogon japonicas.

(ii) Five $(7.7 \%)$ evaluated Huangqi injection manufactured by extracting astragalosides from Radix Astragali.

(iii) Another five (7.7\%) assessed Shexiangbaoxin tablets, which consisted of Moschus, Radix Ginseng, Borneolum Syntheticum, Venenum Bufonis, Cortex Cinnamomi, Calculus Bovis, and Styrax.

(iv) Four (6.2\%) tested Shengmai injection, which is a mixture of extracts from Panax ginseng, Radix Ophiopogonis, and Schisandra chinensis Baill.

(v) Another four (6.2\%) evaluated Tongxinluo capsules, consisting of Radix Ginseng, Scorpio, Hirudo, Eupolyphaga seu Steleophaga, Scolopendra, Periostracum Cicadae, Radix Paeoniae Rubra, and Borneolum Syntheticum.

(vi) Three trials (4.6\%) assessed Shenfu injection, which contains Ginsenoside and Aconitine extracted from Panax ginseng and Aconitum carmichaelii.

(vii) Another three evaluated Suxiao jiuxin pill (4.6\%), consisting of Ligusticum chuanxiong Hort. And Borneolum syntheticum. 







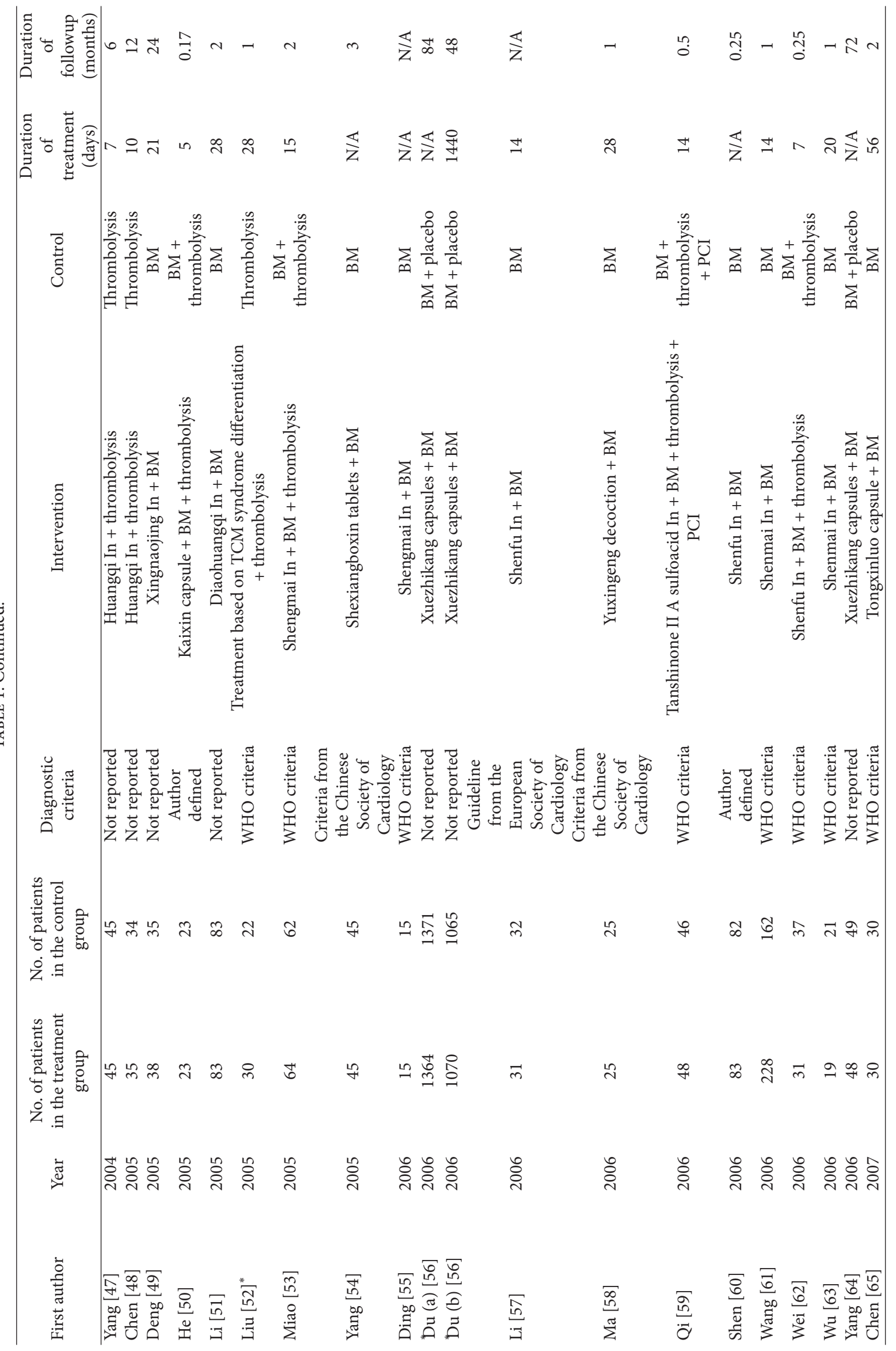




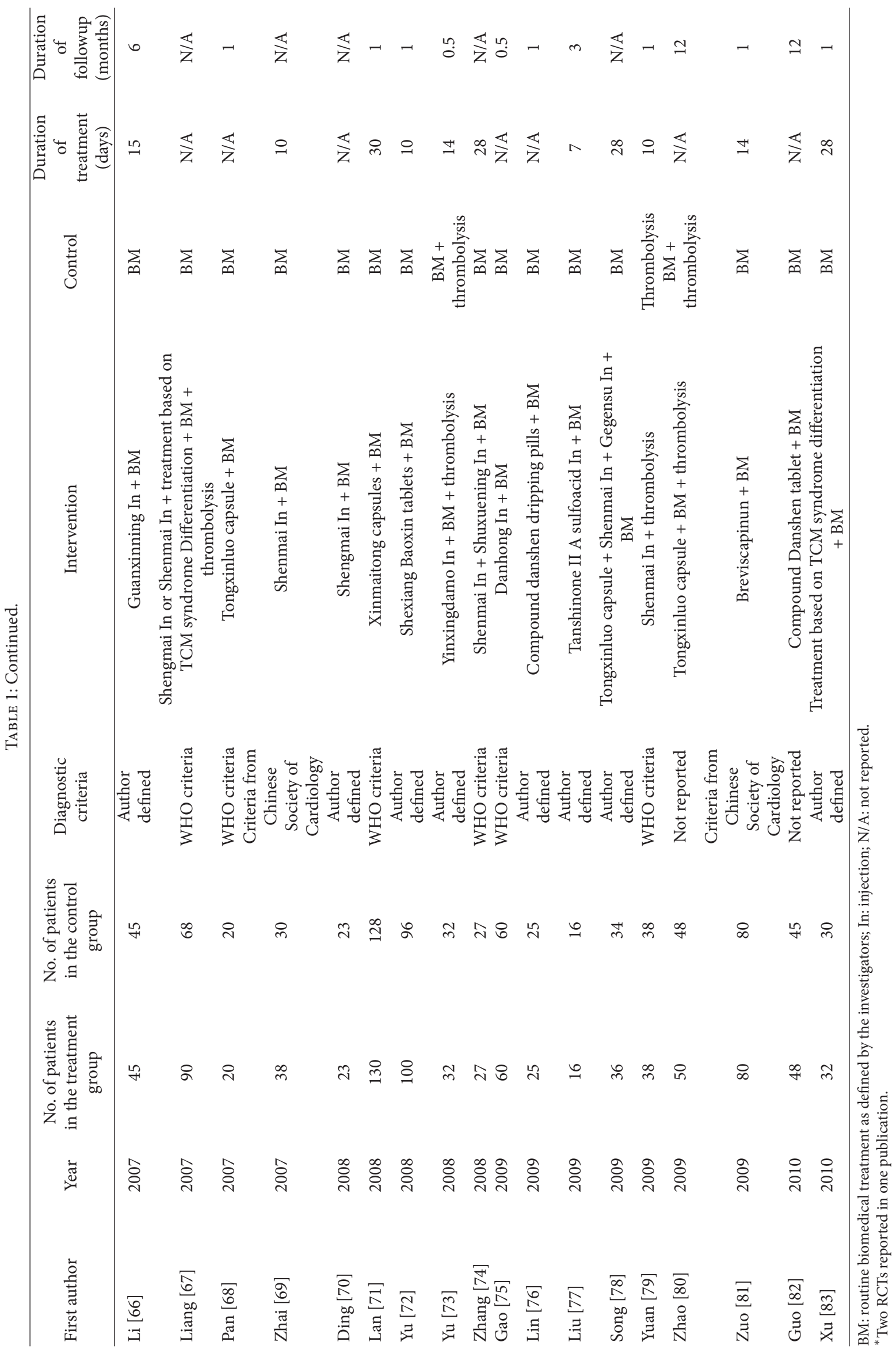




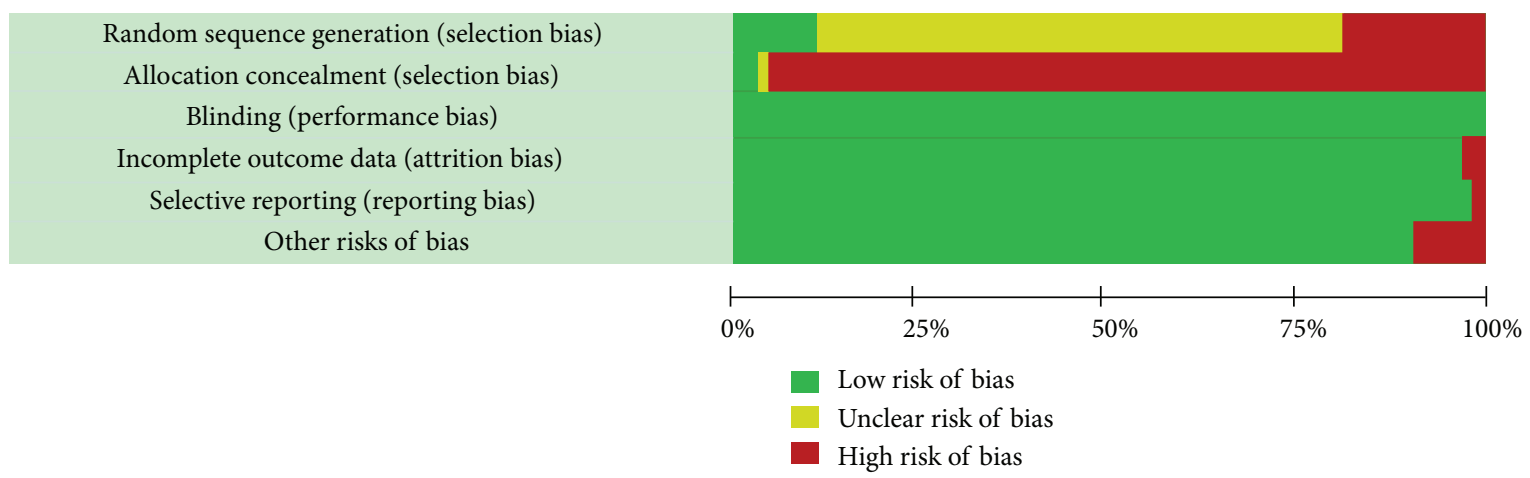

FIGURE 2: Risk of bias amongst included studies: mortality as primary outcome.

(viii) Finally, three (4.6\%) studies tested Xuezhikang capsule, which comprise partially purified extract of fermented Monascus purpureus.

3.3. Risk of Bias. Among these 65 RCTs, only 7 were at low risk for bias for allocation sequence generation. Twelve were at high risk and the remaining RCTs did not report their sequence generation procedure clearly. All but one had high risk of bias in terms of allocation concealment and none of the included studies report the use of blinding. However, we regarded the risks of bias associated with lack of blinding and allocation concealment to be minimal, as the primary outcomes were of objective nature. Two of the included studies had high risk of bias for incomplete data and one for selective outcome reporting. Six are at high risk of bias due to other reasons. In summary, we consider the overall risk of bias amongst our included studies to be moderate (Figure 2 ). The detailed risk of bias assessment results is presented in Appendix 2 in the supplementary materials.

\subsection{Effects of Interventions}

3.4.1. Impact on Fatal Outcomes. In this comparison (Table 2 ), a total of 44 RCTs reported total all-cause mortality. Pooled results demonstrated superiority of combined treatment in preventing all-cause mortality $(\mathrm{RRR}=37 \%, 95 \% \mathrm{CI}=$ $28 \%-45 \%)$. Funnel plot indicates the presence of publication bias. After applying trim and fill procedure (Figure 3), the $\mathrm{RRR}$ remained to be significant $(\mathrm{RRR}=29 \%, 95 \% \mathrm{CI}=$ $16 \%-40 \%$, Table 2). Ten RCTs reported death of cardiac origin, and pooled findings also favor combined treatment $(\mathrm{RRR}=39 \%, 95 \% \mathrm{CI}=22 \%-52 \%)$. Funnel plot indicates the presence of publication bias. After applying trim and fill procedure, the RRR remained to be significant $(R R R=32 \%$, 95\% CI $=15 \%-46 \%$ ).

Pooled results from another four RCTs reporting the occurrence of fatal cardiogenic shock also favored combined treatment $(\mathrm{RRR}=28 \%, 95 \% \mathrm{CI}=5 \%-45 \%)$. Respectively nine, six, five, and three RCTs reported outcomes on sudden cardiac death, fatal myocardial reinfarction, fatal heart failure, and fatal cardiac arrhythmia. In these four comparisons, all pooled findings favored combined treatment (sudden cardiac death: $\mathrm{RRR}=24 \%, 95 \% \mathrm{CI}=6 \%-45 \%$; fatal cardiac reinfarction: $\mathrm{RRR}=54 \%, 95 \% \mathrm{CI}=12 \%-81 \%$; fatal heart failure: $\mathrm{RRR}=52 \%, 95 \% \mathrm{CI}=9 \%-79 \%$; fatal cardiac arrhythmia: $\mathrm{RRR}=29 \%, 95 \% \mathrm{CI}=84 \%-222 \%$ ), but the estimates were statistically insignificant. Except for fatal myocardial reinfarction $\left(I^{2}=37.3 \%\right)$, no significant heterogeneity existed in the comparisons mentioned above. However, given the small number of RCTs reporting this outcome, we were unable to explore heterogeneity using metaregression.

3.4.2. Impact on Nonfatal Cardiovascular Events. In this comparison (Table 2), a total of 11 RCTs reported overall, undifferentiated nonfatal heart events. Pooled results demonstrated superiority of combined treatment in preventing this outcome $(\mathrm{RRR}=48 \%, 95 \% \mathrm{CI}=40 \%-56 \%)$. Twenty-three RCTs evaluated myocardial reinfarction, and the pooled result favors combined treatment $(\mathrm{RRR}=52 \%, 95 \% \mathrm{CI}=$ $39 \%-61 \%)$. The pooled results from 14 and 24 RCTs have also favored combined treatment, respectively, in preventing cardiogenic shock $(\mathrm{RRR}=37 \%, 95 \% \mathrm{CI}=15 \%-53 \%)$ and in alleviating angina symptoms $(\mathrm{RRR}=53 \%, 95 \% \mathrm{CI}=$ 46\%-61\%). Three RCTs investigated nonfatal cardiac rupture as an outcome.The pooled finding supports combined treatment but the estimate was statistically insignificant (RRR $=56 \%, 95 \% \mathrm{CI}=67 \%-89 \%)$. No significant heterogeneity existed in all meta-analyses mentioned above.

Respectively, thirty and twenty-eight RCTs reported outcomes of cardiac arrhythmia and heart failure. In these two groups of studies, pooled findings all favored combined treatment, but high level of heterogeneity existed in both estimates (cardiac arrhythmia: $\mathrm{RRR}=41 \%, 95 \% \mathrm{CI}=27 \%-52 \%, I^{2}=$ $76.2 \%$; heart failure: $\mathrm{RRR}=48 \%, 95 \% \mathrm{CI}=36 \%-58 \%, I^{2}=$ 47.9\%).

3.4.3. Metaregression. We explored these heterogeneities by performing multivariate metaregression analyses using mean age, treatment duration, route of administration (oral versus intravenous), and baseline risk as covariates. None of the four covariates is significantly associated with cardiac arrhythmia (for baseline risk regression coefficient $(\beta)=0.46, P=0.41$; for mean age $\beta=0.00, P=0.96$; for duration of treatment $\beta=0.00, P=0.95$; for route of administration $\beta=0.21, P=$ 0.63 ), or heart failure (for baseline risk $\beta=0.67, P=0.39$; 


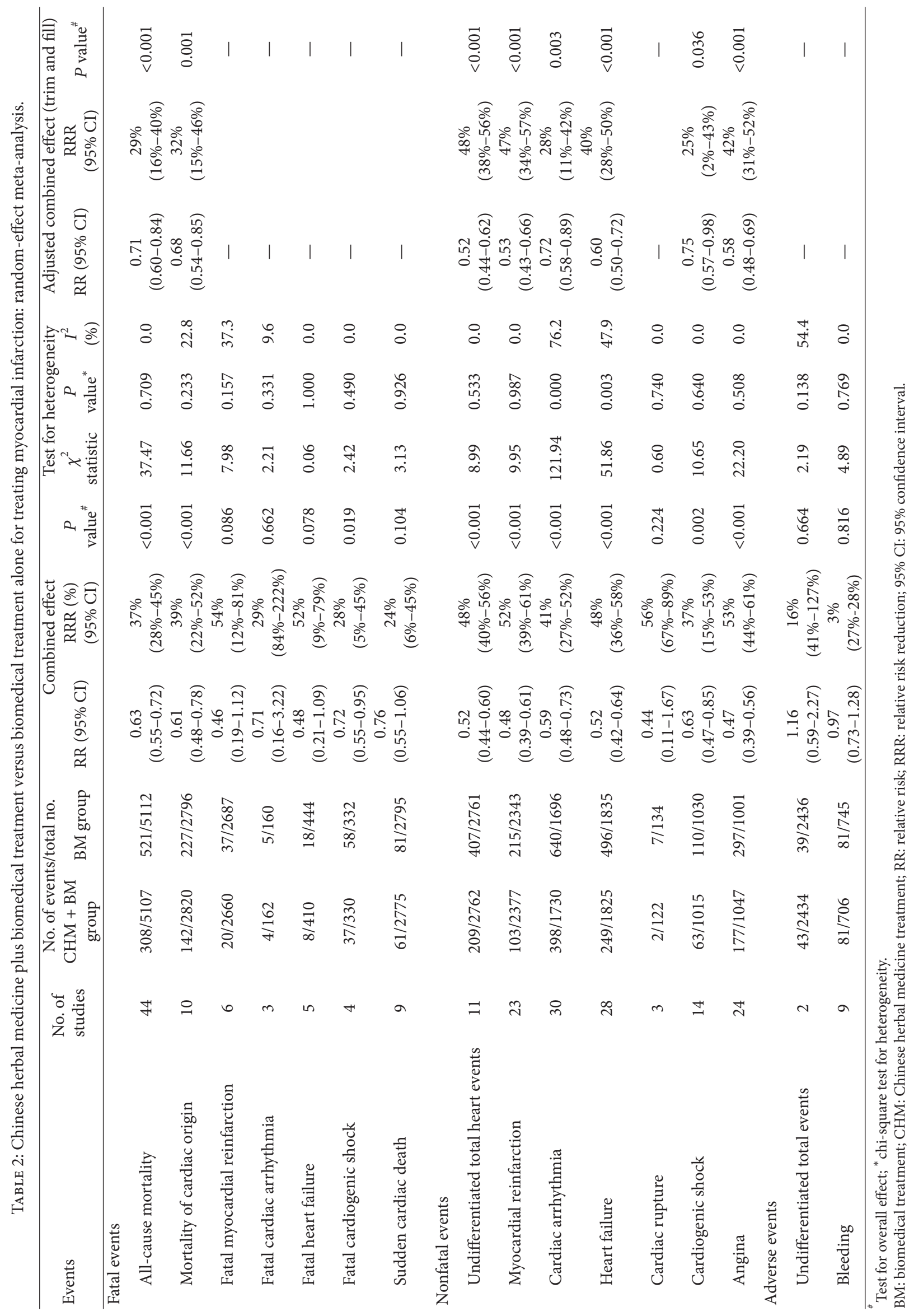




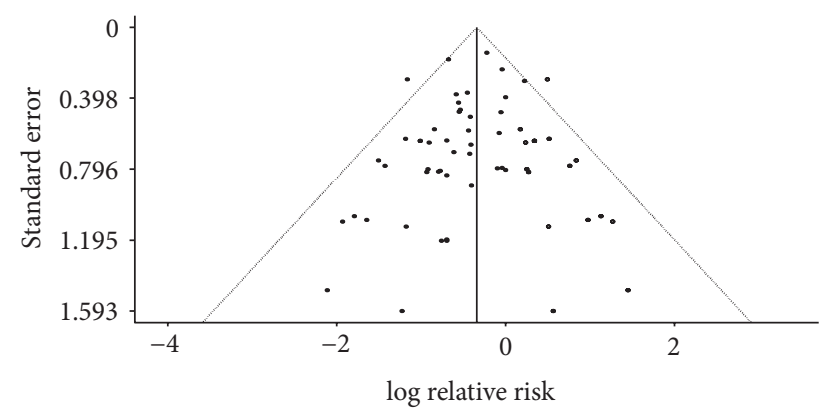

FIgURE 3: Trim and fill funnel plot on the prevention of all-cause mortality.

for mean age $\beta=0.02, P=0.57$; for duration of treatment $\beta=0.00, P=0.87$; for route of administration $\beta=0.12$, $P=0.77)$.

3.4.4. CHM and BM versus BM Alone for MI: Adverse Events. In this comparison (Table 2), nine RCTs reported bleeding as adverse events, but the pooled estimate was statistically insignificant $(\mathrm{RR}=0.97,95 \% \mathrm{CI}=0.73,1.28)$. Two RCTs reported general, undifferentiated adverse events, pooled estimate is heterogeneous and statistically insignificant (RR $\left.=1.16,95 \% \mathrm{CI}=0.59,2.27, I^{2}=54.4 \%\right)$.

\section{Discussion}

This systematic review on the add-on effect of CHM on BM in the treatment of MI summarized findings from 12,022 patients reported in 65 RCTs. The overall risk of bias amongst included studies was moderate. Despite the lack of allocation concealment and blinding in the majority of included trials, its impact on risk of bias was less critical as we focused on objective outcomes. Random-effect meta-analyses demonstrated that combined treatment is superior to BM alone in reducing the risk of all-cause mortality and death of cardiac origin. Funnel plots indicated the presence of publication bias for both outcomes, and trim and fill procedures were conducted as sensitivity analyses. The directions of effect did not change after the adjustment, and the 95\% CI of the estimates overlapped with the unadjusted values. The lower 95\% CI boundary of the trim- and fill-adjusted RRR for allcause and cardiac mortality was $16 \%$ and $15 \%$, respectively. Conservatively speaking, CHM appeared to offer a protective add-on effect against mortality after adjusting for the publication bias, a common problem amongst the clinical research literature on CHM [87].

Combined treatment is also found to be more effective than BM alone in lowering the risk of fatal cardiogenic shock. Our analyses did not demonstrate therapeutic benefits of combined treatment on other reviewed fatal outcomes including myocardial reinfarction, cardiac arrhythmia, heart failure, and sudden cardiac death. For nonfatal outcomes, our analyses demonstrated that CHM is an effective add-on for lowering the risk of cardiogenic shock, cardiac arrhythmia, myocardial reinfarction, and the occurrence of total heart events. Benefits in preventing heart failure and angina were also observed but these findings are less robust given the subjective nature of the outcome, and metaregression did not shed light on potential sources of heterogeneity. We have considered including allocation concealment and blinding as covariates in our metaregressions but numbers of trials with low risk in these domains are too small for conducting such analysis. The effect of combined treatment on these two outcomes would need to be further evaluated with methodologically stronger trials. In addition, more comprehensive reporting on BM treatment details and adverse events is expected in future studies, preferably with reference to the CONSORT statement.

Comprehensiveness of search is the major strength of this systematic review. The use of both international and Chinese databases allowed us to locate a much higher number studies compared to seven existing reviews on the topic [88]. We also attempted to synthesize results from trials evaluating heterogeneous CHM using random-effect model. This allowed us to estimate the average effect of adding CHM on top of conventional therapies [12]. The use of the trim and fill method has also partly circumvented the problem of publication bias. Nevertheless, the robustness of our conclusion depends on the assumption that the objective nature of outcomes was less affected by two major sources of bias: allocation concealment and blinding. While this assumption is tested in metaepidemiological studies [89, 90], the generalizability of these findings warrants further investigations.

\section{Conclusion}

Based on RCTs of moderate quality, this systematic review demonstrated consistent, add-on benefits of using CHM on top in BM treatment for preventing all-cause and cardiac mortality amongst MI patients. These findings are in line with the results from seven existing systematic reviews of smaller scope and lower methodological quality. This tentative conclusion warrants further scrutiny using rigorously designed $\mathrm{RCT}$, and a more comprehensive approach in reporting BM treatment details and adverse events is warranted.

\section{Authors' Contribution}

V. C. H. Chung and M. Chen are the cofirst authors of this paper.

\section{Acknowledgment}

This paper is funded by the Chinese Medicine Department, Hong Kong Hospital Authority (CUHK Project code: 7050098).

\section{References}

[1] A. P. Wakai, "Myocardial infarction (ST-elevation)," BMJ Clinical Evidence, no. 202, article 01, 2011.

[2] A. D. Lopez, C. D. Mathers, M. Ezzati, D. T. Jamison, and C. J. Murray, "Global and regional burden of disease and risk factors, 
2001: systematic analysis of population health data," Lancet, vol. 367, no. 9524, pp. 1747-1757, 2006.

[3] S. Unverzagt, K. Hirsch, M. Buerke et al., "Inotropic agents and vasodilator strategies for acute myocardial infarction complicated by cardiogenic shock or low cardiac output syndrome (Protocol)," Cochrane Database of Systematic Reviews, no. 2, Article ID CD009669, 2012.

[4] J. L. Tang, B. Y. Liu, and K. W. Ma, "Traditional Chinese medicine," The Lancet, vol. 372, no. 9654, pp. 1938-1940, 2008.

[5] J. Xu and Y. Yang, "Traditional Chinese medicine in the Chinese health care system," Health Policy, vol. 90, no. 2-3, pp. 133-139, 2009.

[6] H. Shang, J. Chen, J. Zhang et al., “Three therapeutic tendencies for secondary prevention of myocardial infarction and possible role of Chinese traditional patent medicine: viewpoint of evidence-based medicine," Journal of Evidence-Based Medicine, vol. 2, no. 2, pp. 84-91, 2009.

[7] L. Zhang, Y. Yang, Y. Wang, and X. Gao, "Astragalus membranaceus extract promotes neovascularisation by VEGF pathway in rat model of ischemic injury," Pharmazie, vol. 66, no. 2, pp. 144-150, 2011.

[8] P. Jiang, W. Dai, S. Yan et al., "Biomarkers in the early period of acute myocardial infarction in rat serum and protective effects of Shexiang Baoxin Pill using a metabolomic method," Journal of Ethnopharmacology, vol. 138, no. 2, pp. 530-536, 2011.

[9] Y. T. Cheng, Y. J. Yang, H. T. Zhang et al., "Pretreatment with Tongxinluo protects porcine myocardium from ischaemia/reperfusion injury through a nitric oxide related mechanism," Chinese Medical Journal, vol. 122, no. 13, pp. 1529-1538, 2009.

[10] N. Xu, C. Qiu, W. Wang et al., "HPLC/MS/MS for quantification of two types of neurotransmitters in rat brain and application: myocardial ischemia and protection of Sheng-Mai-San," Journal of Pharmaceutical and Biomedical Analysis, vol. 55, no. 1, pp. 101-108, 2011.

[11] N. Wang, S. Minatoguchi, Y. Uno et al., "Treatment with shengmai-san reduces myocardial infarct size through activation of protein kinase C and opening of mitochondrial KATP channel," American Journal of Chinese Medicine, vol. 29, no. 2, pp. 367-375, 2001.

[12] R. D. Riley, J. P. Higgins, and J. J. Deeks, "Interpretation of random effects meta-analyses," British Medical Journal, vol. 342, article d549, 2011.

[13] A. Shang, K. Huwiler, L. Nartey, P. Jüni, and M. Egger, "Placebo-controlled trials of Chinese herbal medicine and conventional medicine-Comparative study," International Journal of Epidemiology, vol. 36, no. 5, pp. 1086-1092, 2007.

[14] L. Wood, M. Egger, L. L. Gluud et al., "Empirical evidence of bias in treatment effect estimates in controlled trials with different interventions and outcomes: meta-epidemiological study," British Medical Journal, vol. 336, no. 7644, pp. 601-605, 2008.

[15] A. Hrobjartsson, A. S. Thomsen, F. Emanuelsson et al., "Observer bias in randomised clinical trials with binary outcomes: systematic review of trials with both blinded and nonblinded outcome assessors," British Medical Journal, vol. 344, article e1119, 2012.

[16] "Chinese pharmacopoeia online," Chinese Pharmacopoeia Comission, http://www.chp.org.cn/cms/home/.

[17] J. P. T. Higgins, D. G. Altman, P. C. Gøtzsche et al., "The Cochrane Collaboration's tool for assessing risk of bias in randomised trials," British Medical Journal, vol. 343, article d5928, 2011.

[18] J. L. Peters, A. J. Sutton, D. R. Jones, K. R. Abrams, and L. Rushton, "Performance of the trim and fill method in the presence of publication bias and between-study heterogeneity," Statistics in Medicine, vol. 26, no. 25, pp. 4544-4562, 2007.

[19] J. P. T. Higgins, S. G. Thompson, J. J. Deeks, and D. G. Altman, "Measuring inconsistency in meta-analyses," British Medical Journal, vol. 327, no. 7414, pp. 557-560, 2003.

[20] "Investigating heterogeneity," in Cochrane Handbook for Systematic Reviews of Interventions, J. P. T. Higgins and S. Green, Eds., chapter 9.6, The Cochrane Collaboration, 2011.

[21] CHD Group of Guang'anmen Hospital, "Comparison of treatments with combined Traditional Chinese and western medicine or western medicine alone for acute myocardial infarction: 276 cases analysis," Chinese Journal of Integrative Medicine, vol. 1, no. 1, pp. 10-12, 1981.

[22] W. Kou, Z. Chen, S. Tao et al., "A randomized controlled trials on 268 patients with acute myocardial infarction treated with Yiqihuoxue injection," Chinese Journal of Integrative Medicine, vol. 3, no. 3, pp. 146-148, 1983.

[23] K. Chen, Z. Qian, and Q. Dong, "A randomized controlled trials on 224 patients with acute myocardial infarction treated with Yiqihuoxue injection," Chinese Journal of Integrative Medicine, vol. 4, no. 7, article 416, 1984.

[24] S. Liang, "Clinical observation of dehydrocorydalineon acute myocardial infarction," Tianjin Journal of Traditional Chinese Medicine, no. 4, pp. 3-7, 1989.

[25] X. Xia, "Combined Dushentang and Thrombolytic therapy in the treatment of acute myocardial infarction," Journal of Jiamusi Medical College, vol. 16, no. 2, pp. 32-33, 1993.

[26] C. Li and S. Zhang, "Combined traditional Chinese and western medicine in the treatment of acute myocardial infarction with cardiogenic shock," Chinese Critical Care Medicine, vol. 6, no. 6, pp. 355-358, 1994.

[27] S. Li and F. Li, "Clinical observation of Huangqi and Glucoseinsulin-potassium on 18 patients with acute myocardial infarction," Chinese Journal of Integrative Medicine, vol. 14, no. 6, article 359, 1994.

[28] G. Yang, Q. Pang, Y. Peng, K. Wang, and S. Xing, "Influence of Shexiang Baoxin Wan on survival rate after acute myocardial infarction," Shanghai Medical \& Pharmaceutical Journal, no. 8, pp. 21-22, 1997.

[29] R. Zhang, C. Tu, Y. Yang, Y. Zhao, and X. Wu, "Tradition Chinese medicine (TCM) diagnostic system and clinical observation of Jianxinpian on myocardial infarction," Tianjin Journal of Traditional Chinese Medicine, vol. 15, no. 4, pp. 160-162, 1998.

[30] S. Guo and Y. Zhang, "Clinical Study on the therapeutic effect of shenmai injection against myocardium stunning in thrombolytic therapy with urokinase for acute myocardialnfarction," Acta Academiae Medicinae Weifang, vol. 21, no. 4, pp. 271-273, 1999.

[31] G. Li, W. Qi, K. Xiong, and X. G. H. Yang, "Clinical observation on 51 patients of acute myocardial infarction treated with thrombolytic therapy combined with Chinese herbal medicine," Chinese Journal of Integrative Medicine, vol. 19, no. 8, pp. 461-462, 1999.

[32] D. Zhang, B. Tang, N. Xu, R. Yang, B. Chang, and J. Yan, "Clinical observation of combined traditional Chinese and western medicine on 52 patients with acute myocardial infarction," 
Beijing Journal of Traditional Chinese Medicine, vol. 18, no. 5, pp. 16-17, 1999.

[33] S. Guo and Y. Zhang, "Clinical study on the therapeutic effect of suxiaojiuxinwan against myocardium stunning in thrombolytic therapy with urokinase for acute myocardial infarction," Tianjin Pharmacy, vol. 12, pp. 1-3, 2000.

[34] G. Han, Z. Ma, X. Xue et al., "Influences of astragulas injection on reperfusion injury of intravenous thrombolytic therapy in patients with acute myocardial infarction," Chinese Journal of Integrated Traditional and Western Medicine in Intensive and Critical Care, vol. 7, no. 2, pp. 107-108, 2000.

[35] J. Li and B. Chen, "Effect of Zhubitang in acute myocardial infarction," Journal of Emergency in Traditional Chinese Medicine, vol. 9, no. 2, pp. 50-51, 2000.

[36] Q. Li, C. Huang, X. Pan, and Z. Li, "Influence of SuxiaojiuxinWan on survival rate after acute myocardial infarction," Tianjin Pharmacy, vol. 12, pp. 12-13, 2000.

[37] J. Lu, Y. Chen, J. Liang, Q. Yang, C. Li, and X. Yang, "Clinical observation on treatment with leech injection for acute myocardial infarction," Chinese Journal of Integrated Traditional and Western Medicine in Intensive and Critical Care, vol. 7, no. 3, pp. 152-154, 2000.

[38] K. Yin, Y. Luo, T. Liu, X. Zou, and G. Mei, "Clinical observation of combined intravenous thrombolytic therapy and traditional Chinese medicine on 28 patients with acute myocardial infarction," Journal of Emergency in Traditional Chinese Medicine, vol. 9, no. 3, pp. 89-90, 2000.

[39] G. Wu, N. Li, S. Bai, and J. Zhang, "Study on the treatment effect of Huangqi Danshen injection in treating acute myocardial infarction," Journal of Clinical Cardiology, vol. 17, no. 2, article 91, 2001.

[40] Y. Bai, Y. Wang, Y. Hao, Y. Liang, X. Li, and B. Qian, "Clinical observation of 999 Shenmai injection and western medicine on 62 patients with acute myocardial infarction," Modern Journal of Integrated Traditional Chinese and Western Medicine, vol. 11, no. 4, pp. 285-286, 2002.

[41] J. Shi, X. Zhang, and W. Sun, "Complementary effect of Dengzhanhuasu on acute myocardial infarction," Chinese Journal of Integrative Medicine, vol. 22, no. 3, pp. 227-228, 2002.

[42] Y. Guan and J. Guan, "Influence of Xingding injection on the prognosis after acute myocardial infarction and hemorrheology," Liaoning Journal of Traditional Chinese Medicine, vol. 30, no. 2, article 122, 2003.

[43] S. Zhang, Y. Liu, and X. Wang, "Combined traditional Chinese and western medicine on 45 patients with acute myocardial infarction," Journal of Practical Traditional Chinese Internal Medicine, vol. 17, no. 3, article 186, 2003.

[44] G. Han, T. Wang, Z. Bai, Q. Wang, and B. Yang, "Influence of Shexiang Baoxin Wan on reperfusion injury after thrombolytic therapy in acute myocardial infarction," Chinese Traditional Patent Medicine, vol. 26, pp. 30-31, 2004.

[45] X. Li, W. Zhang, Z. Wang et al., "Clinical observation of Shexiang Baoxin Wan on acute myocardial infarction," Chinese Traditional Patent Medicine, vol. 26, pp. 32-34, 2004.

[46] S. Liu, Y. Liu, and Y. Wang, "Study of effect of Shenmai injection on acute myocardial infarction," China Journal of Modern Medicine, vol. 14, no. 5, pp. 108-109, 2004.

[47] G. Yang, C. Li, and A. Tang, "Study on treatment effect of astragalus injection combined thrombolytic therapy on ischemia/reperfusion injury in acute myocardial infarction," Chinese Journal of Integrated Traditional and Western Medicine in Intensive and Critical Care, vol. 11, no. 3, pp. 162-164, 2004.
[48] L. Chen, H. Zhang, X. Zheng, and A. Wang, "Influence of Huangqi injection on cardiovascular events after thrombolytic therapy," Chinese Journal of Integrative Medicine, vol. 14, no. 10, article 1288, 2005.

[49] Y. Deng, X. Chen, and J. Liu, "Clinical observation of Xingnaojing injection on acute myocardial infarction," Journal of Emergency in Traditional Chinese Medicine, vol. 14, no. 12, pp. 1179-1180, 2005.

[50] Y. He, J. Chen, and J. Liang, "The clinical observation on kaixin capsule for ischemical reperfusion injury after acute myocardial infarction, the syndrome of blood stasis due to deficiency of Qi in TCM," Journal of Emergency in Traditional Chinese Medicine, vol. 14, no. 12, pp. 1137-1139, 2005.

[51] H. Li, "Anti-myocardial ischemia effect of Di'aohuangqi injection in acute myocardial infarction," Chinese Journal of Practical Medicine, vol. 32, no. 7, article 49, 2005.

[52] Y. Liu, "Combined thrombolytic therapy and tradition Chinese medicine (TCM) syndrome differentiation in the treatment of acute myocardial infarction," Modern Journal of Integrated Traditional Chinese and Western Medicine, vol. 14, no. 6, pp. 733-734, 2005.

[53] Y. Miao, F. Chen, and M. Ma, "Prevention effect of Shengmai injection in reperfusion injury after urokinase thrombolytic therapy on patients with acute myocardial infarction," Chinese Medicine of Factory and Mine, vol. 18, no. 4, pp. 363-364, 2005.

[54] Y. Yang, Y. Ye, and L. Mao, "Influence of Shexiang Baoxin Wan on ventricular structure and function after acute myocardial infarction," Journal of the Practical Chinese With Modern Medicine, vol. 18, no. 11, pp. 1583-1584, 2005.

[55] L. Ding and M. Xu, "The complementary effect of Shenmai injection in treating acute myocardial infarction complicated by cardiogenic shock Gansu," Journal of Traditional Chinese Medicine, vol. 19, no. 8, article 11, 2006.

[56] B. M. Du, Z. L. Lu, Z. Chen, Y. F. Wu, W. D. Zhao, and T. G. Huang, "China coronary secondary prevention study: analysis of patients with different myocardial infarction history," Chinese Journal of Internal Medicine, vol. 45, no. 1, pp. 21-24, 2006.

[57] Z. E. Li, "Clinical research on effects of shenfu injection in different dosage in preventing heart failure occurred in patients of acute myocardial infarction with elevated ST segment," Chinese Journal of Integrative Medicine, vol. 26, no. 6, pp. 555-557, 2006.

[58] L. Ma, D. Shi, J. Liu, L. Ma, X. Zhu, and K. Meng, "Influence of Yuxingeng fluid on left ventricular function after acute myocardiac infarction," Chinese Journal of Integrative Medicine, vol. 26, no. 2, pp. 167-168, 2006.

[59] H. Qi, X. Zhao, and Y. Li, “The effect of Sodium tanshinone IIA sulfonate (STS) injection in treating acute myocardiac infarction (AMI)," China Medical Herald, vol. 3, no. 23, pp. 22-24, 2006.

[60] Y. Shen, H. Gao, and G. Ruan, "Clinical observation of Shenfu injection on acute myocardiac infarction," Journal of Qiqihar Medical College, vol. 27, no. 9, pp. 1038-1039, 2006.

[61] S. Wang and Y. Guo, "Clinical observation of Shenmai injection on acute myocardiac infarction," Chinese Journal of Information on Traditional Chinese Medicine, vol. 13, no. 6, pp. 64-65, 2006.

[62] X. Wei, "Clinical observation of combined thrombolytic therapy and Shenfu injection for ischemical reperfusion injury," Journal of Emergency in Traditional Chinese Medicine, vol. 15, no. 8, pp. 866-867, 2006.

[63] J. Wu and W. Wu, "Clinical observation of shenmai injection for reperfusion injury after intravenous thrombolysis on 30 
patients with acute myocardial infarction," Nei Mongol Journal of Traditional Chinese Medicine, vol. 25, no. 3, article 8, 2006.

[64] J. Yang, J. Li, L. Zhang, L. Song, and P. Han, "Secondary prevention effect of xuezhikang on coronary heart disease," Chinese Circulation Journal, vol. 21, no. 4, pp. 276-278, 2006.

[65] H. Chen, S. Cai, C. Hong, X. Liu, and Z. Wong, "Clinical observation of Tongxinluo capsule on acute myocardiac infarction," Journal of Emergency in Traditional Chinese Medicine, vol. 16, no. 7, pp. 823-824, 2007.

[66] Z. Li, W. Zheng, and $\mathrm{H}$. Wu, "Influence of Guanxinning injection on left ventricular remodeling after acute myocardial infarction," Chinese Journal of Integrated Traditional and Western Medicine in Intensive and Critical Care, vol. 14, no. 4, pp. 227-230, 2007.

[67] C. Liang, "Clinical observation on 90 patient of acute myocardiac infarction treated with combined traditional Chinese and western medicine," Journal of Practical Traditional Chinese Internal Medicine, vol. 21, no. 6, pp. 55-56, 2007.

[68] G. Pan, F. Xu, X. Xu, X. Feng, and Z. Hu, "Clinic observation of tongxinluo capsule in conservative treatment of patients with AMI," Progress in Modern Biomedicine, vol. 7, no. 6, article 957, 2007.

[69] Y. Zhai, "Effect of Shenmai injection on QT dispersion in patients with acute myocardiac infarction," Chinese Journal of Integrative Medicine, vol. 16, no. 7, pp. 916-917, 2007.

[70] Y. Ding and W. Dong, "Clinical observation of Shenmai injection in the treatment of elder patients with acute myocardiac infarction," Chinese Community Doctors, vol. 10, no. 23, article 129, 2008.

[71] J. Lan, "The influence of Xinmaitong capsule on heart rate variability in patients with acute myocardiac infarction," Chinese Journal of the Practical Chinese With Modern Medicine, vol. 21, no. 14, pp. 1187-1189, 2008.

[72] C. Yu and C. Li, "Clinical observation on angina pectoris after intravenous thrombolysis in acute myocardial infarction treated with combination of TCM and western medicine," Journal of Practical Traditional Chinese Medicine, vol. 24, no. 9, pp. 579-580, 2008.

[73] T. Yu and M. Zhu, "Clinical observation on 32 patients of acute myocardiac infarction treated with combination of Yinxingdamo injection and intravenous thrombolytic therapy," Journal of New Chinese Medicine, vol. 40, no. 10, pp. 41-43, 2008.

[74] J. Zhang, "Clinical observation of Shuxuening and Shenmai injection on acute myocardiac infarction," China Medical Herald, vol. 5, no. 8, pp. 68-69, 2008.

[75] Z. Gao, "Clinical Effects of Danhong Injecta on complications of Acute Myocardial Infarction," China Medical Herald, vol. 11, no. 4, article 582, 2009.

[76] H. Lin, I. Yang, and D. Zheng, "Clinical observation on the routine and Compound Danshen Dropping Pills in the treatment of patients with acute myocardial infarction," China Medical Herald, vol. 6, no. 25, pp. 52-53, 2009.

[77] P. Liu, Y. Zhang, J. Tang, and H. Li, “The effect and clinical significance of tanshinone injection on plasma NT-proBNP in acute myocardial infarction," Chinese Journal of Difficult and Complicated Cases, vol. 8, no. 6, pp. 327-329, 2009.

[78] X. Song, N. Gao, and J. Zou, "Thirty-six cases of acute inferior myocardial infarction complicated by right ventricular infarction by integrative treatment," Chinese Journal of Integrative Medicine on Cardio-/Cerebrovascular Disease, vol. 7, no. 5, pp. 516-517, 2009.
[79] X. Yuan, "Clinical observation of combined intravenous thrombolytic therapy and Shenmai injection on acute myocardiac infarction treated," China Modern Medicine, vol. 16, no. 10, article 56, 2009.

[80] Q. Zhao and Q. Wei, “The complementary effect of Tongxinluo capsule on acute myocardiac infarction," Chinese Journal of Integrative Medicine, vol. 18, no. 13, article 1507, 2009.

[81] Y. Zuo, R. Jia, and D. Yu, "The influence and therapeutic effect of Dengzhanhuasu on the high-sensitivity C-reactive protein of patients with acute myocardial infarction," Clinical Medicine of China, vol. 25, no. 3, pp. 291-293, 2009.

[82] H. Guo, "Clinical observation on anti-myocardial ischemia activity after acute myocardiac infarction treated with Fufangdanshenpian," Chinese Journal of Ethnomedicine and Ethnopharmacy, no. 1, article 142, 2010.

[83] Q. Xu and Q. Xiong, "Combined traditional Chinese and western medicine in the treatment of acute myocardiac infarction," Hubei Journal of Traditional Chinese Medicine, vol. 32, no. 3, pp. 20-22, 2010.

[84] E. Rapaport, R. Bernard, and E. Corday, "Nomenclature and criteria for diagnosis of ischemic heart disease. Report of the joint International Society and Federation of Cardiology/World Health Organization Task Force on standardization of clinical nomenclature," Circulation, vol. 59, no. 3, pp. 607-609, 1979.

[85] Chinese Society of Cardiology, "AMI guidelines for diagnosis and treatment," Chinese Journal of Cardiology, vol. 29, no. 12, pp. 710-725, 2001.

[86] F. Van De Werf, D. Ardissino, A. Betriu et al., "Treatment of acute myocardial infarction in patients with ST segment elevation at onset of symptoms: the task force on the management of acute myocardial infarction of the European Society of Cardiology," Italian Heart Journal Supplement, vol. 4, no. 12, pp. 981-1016, 2003.

[87] A. Shang, K. Huwiler, L. Nartey, P. Jüni, and M. Egger, "Placebo-controlled trials of Chinese herbal medicine and conventional medicine-Comparative Study," International Journal of Epidemiology, vol. 36, no. 5, pp. 1086-1092, 2007.

[88] J. Luo and H. Xu, "Outcome measures of Chinese Herbal Medicine for Coronary Heart Disease: an overview of systematic reviews," Evidence-Based Complementary and Alternative Medicine, vol. 2012, Article ID 927392, 9 pages, 2012.

[89] L. Wood, M. Egger, L. L. Gluud et al., "Empirical evidence of bias in treatment effect estimates in controlled trials with different interventions and outcomes: meta-epidemiological study," British Medical Journal, vol. 336, no. 7644, pp. 601-605, 2008.

[90] A. Hróbjartsson, A. S. S. Thomsen, F. Emanuelsson et al., "Observer bias in randomised clinical trials with binary outcomes: systematic review of trials with both blinded and nonblinded outcome assessors," British Medical Journal, vol. 344, article e1119, 2012. 


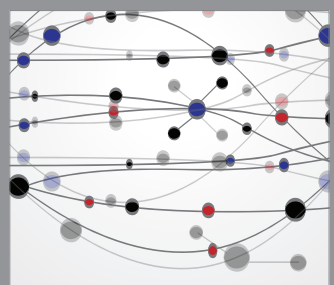

The Scientific World Journal
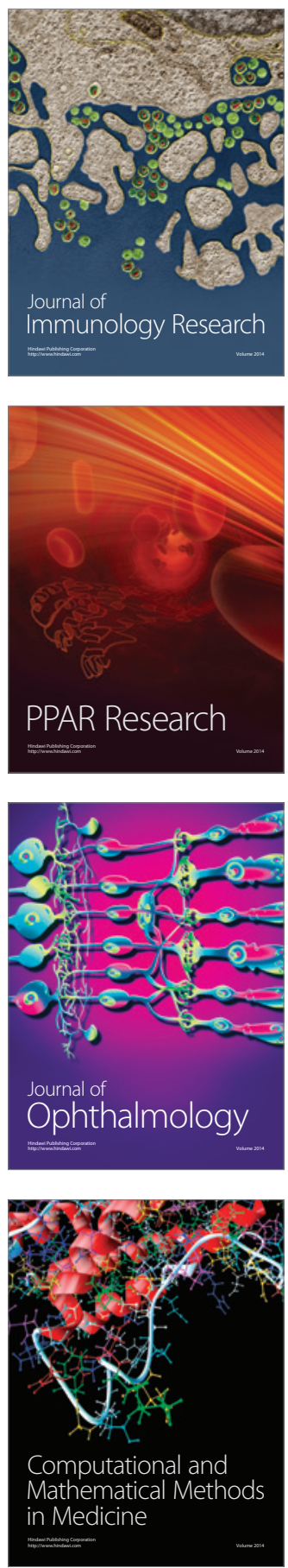

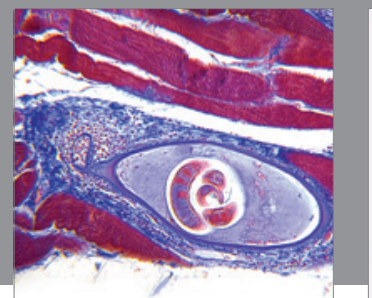

Gastroenterology

Research and Practice
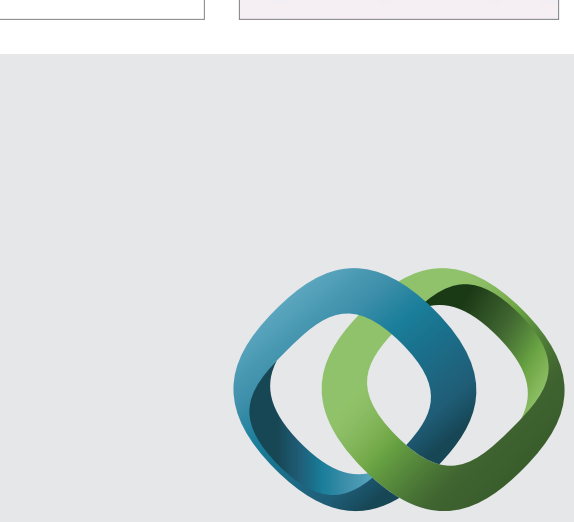

\section{Hindawi}

Submit your manuscripts at

http://www.hindawi.com
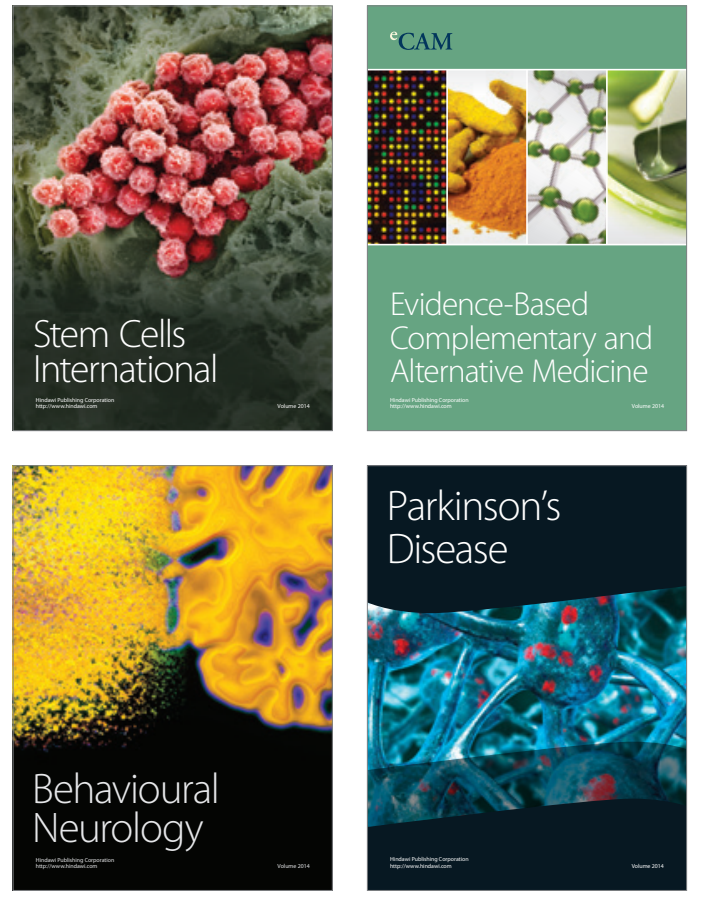
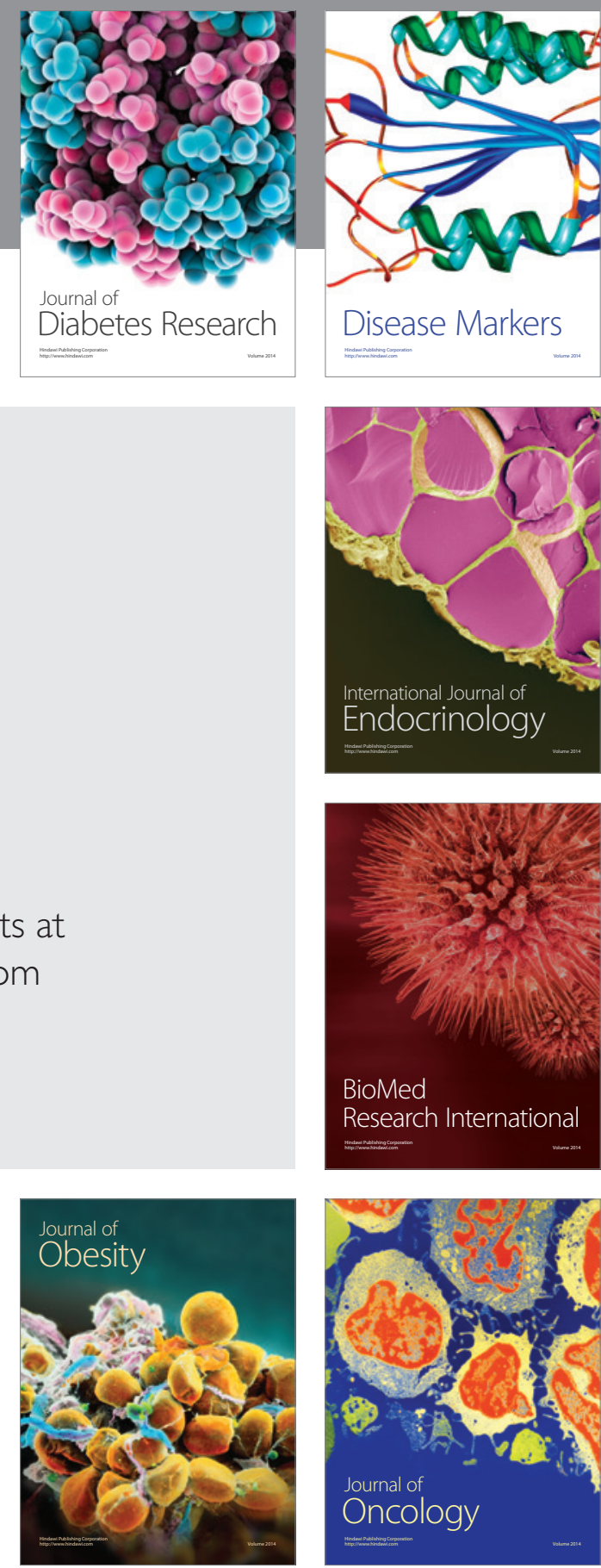

Disease Markers
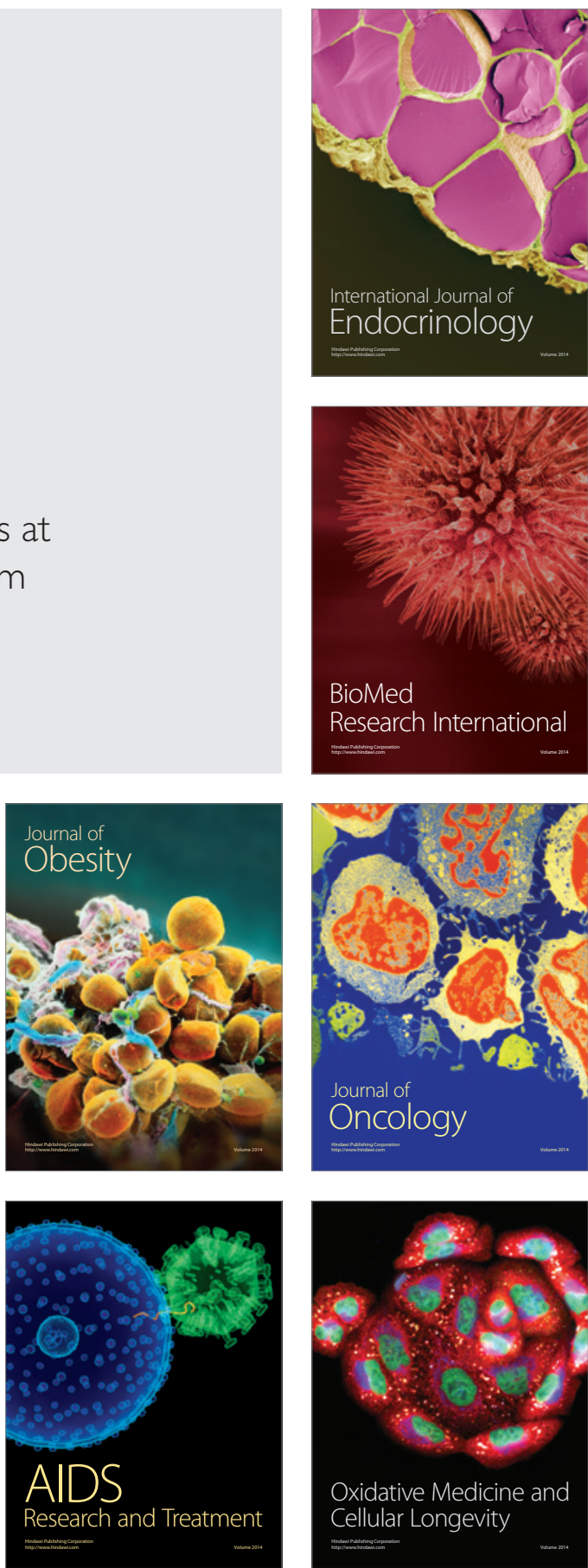